OPEN ACCESS

Edited by:

Sead Sabanadzovic

Mississippi State University,

United States

Reviewed by:

Sotaro Chiba,

Nagoya University Asian Satellite

Campuses Institute, Japan

Eeva Johanna Vainio,

Natural Resources Institute Finland,

Finland

*Correspondence:

Zhensheng Kang

kangzs@nwsuaf.edu.cn

Specialty section:

This article was submitted to

Virology,

a section of the journal

Frontiers in Microbiology

Received: 25 March 2017 Accepted: 22 September 2017 Published: 10 October 2017

Citation:

Zheng L, Lu X, Liang $X$, Jiang $S$,

Zhao J, Zhan G, Liu P, Wu J and

Kang Z (2017) Molecular

Characterization of Novel

Totivirus-Like Double-Stranded RNAs from Puccinia striiformis $f$. sp. tritici, the Causal Agent of Wheat Stripe

Rust. Front. Microbiol. 8:1960.

doi: 10.3389/fmicb.2017.01960

\section{Molecular Characterization of Novel Totivirus-Like Double-Stranded RNAs from Puccinia striiformis f. sp. tritici, the Causal Agent of Wheat Stripe Rust}

\author{
Li Zheng, Xia Lu, Xiaofei Liang, Shuchang Jiang, Jing Zhao, Gangming Zhan, Peng Liu, \\ Jianhui Wu and Zhensheng Kang*
}

State Key Laboratory of Crop Stress Biology for Arid Areas and College of Plant Protection, Northwest A\&F University Yangling, China

Characterization of newly isolated mycoviruses may contribute to understanding of the evolution and diversity of viruses. Here, a deep sequencing approach was used to analyze the double-stranded RNA (dsRNA) mycoviruses isolated from field-collected $P$. striiformis samples in China. Database searches showed the presence of at least four totivirus-like sequences, termed Puccinia striiformis virus 1 to 4 (PsV1 to 4). All of these identified sequences contained two overlapping open reading frames (ORFs) which encode a putative coat protein (CP) and an RNA-dependent RNA polymerase (RdRp) showing similar structures to members of the genus Totivirus. Each PsV contained a -1 ribosomal frameshifting region with a slippery site and a pseudoknot structure in the overlapped regions of these ORFs, indicating that the RdRp is translated as a CPRdRp fusion. Phylogenetic analyses based on RdRp and CP suggested that these novel viruses belong to the genus Totivirus in the family Totiviridae. The presences of these PsVs were further validated by transmission electron microscope (TEM) and RT-PCR. Taken together, our results demonstrate the presence of diverse, novel totiviruses in the P. striiformis field populations.

Keywords: wheat stripe rust, Puccinia striiformis, deep sequencing, mycovirus, Totivirus

\section{INTRODUCTION}

Mycoviruses (fungal viruses) are of common occurrence in all major taxonomic groups of filamentous fungi, yeasts and oomycetes (Pearson et al., 2009; Ghabrial et al., 2015). Although most mycoviruses infect their fungal hosts symptomlessly (cryptic infections), some of them cause phenotypic alterations including hypovirulence and debilitation, and thus can be used as biological control agents against fungal diseases, such as the +ssRNA mycovirus Cryphonectria hypovirus 1 (CHV1) against Cryphonectia parasitica (Nuss, 2005) and the ssDNA mycovirus Sclerotinia sclerotiorum hypovirulence-associated DNA virus 1 (SsHADV-1) against S. sclerotiorum (Yu et al., 2013). Most mycoviruses have double-stranded RNA (dsRNA) genomes and form typical virus particles (Lin et al., 2012; Zheng et al., 2014). DsRNA mycoviruses are now classified into six families, including Totiviridae, Partitiviridae, Megabirnaviridae, Reoviridae, Quadriviridae, 
Chrysoviridae (Kondo et al., 2016). Among them, the families Totiviridae and Partitiviridae are the largest.

The Basidiomycota rust fungi cause disease on a variety of host crop species including soybean, coffee, groundnuts, wheat, and tree species such as conifers and poplars. Rust fungi belong to the order Pucciniales which consists of over 5,000 species and over 100 genera (Zhang et al., 1994). Rusts are obligate biotrophs, which can only absorb nutrients from alive host tissue. Wheat stripe rust, caused by Puccinia striiformis f. sp. tritici, is one of the most important diseases of wheat worldwide (Chen, 2005; Wellings, 2011). In China, the annual yield loss was estimated to be approximately 1.0 million metric tons (Chen et al., 2009). The presence of dsRNA in rusts was first reported in Newton et al. (1985). Subsequently, indirect evidence suggested the presence of mycovirus-like RNA molecules in Phakopsora pachyrhizi, the causal agent of Asian soybean rust (Link et al., 2014). Recently, a dsRNA mycovirus was identified from the fungus $P$. pachyrhizi (Cooper et al., 2016). Although some progress has been made in obtaining dsRNAs from rust mycoviruses, little is known about their genome organization, which is due in a large extent to their asymptomatic infections (Zhang et al., 1994). The cryptic dsRNAs are mostly reported in the families Totiviridae and Partitiviridae (Ghabrial, 1998; Zheng et al., 2014). The family Totiviridae currently comprises five approved genera, of which totiviruses and victoriviruses infect only fungi, while giardiaviruses, trichomonasviruses and leishmaniaviruses infect mainly protozoa (Goodman et al., 2011; Kondo et al., 2016). Members of this family have nonsegmented dsRNA genomes being 4.6-7.0 kbp in length and usually contain two large, partially overlapping open reading frames (ORFs) which encode a capsid protein (CP) and an RNAdependent RNA polymerase (RdRp) respectively (Ghabrial et al., 2015).

Here we report four new complete totivirus-like genome sequences based on deep sequencing of dsRNAs isolated from field-collected $P$. striiformis samples in China. Based on viral genome organization, phylogeny and particle morphology, these novel viruses were identified to belong to the genus Totivirus in the family Totiviridae.

\section{MATERIALS AND METHODS}

\section{P. striiformis Fungal Samples}

Puccinia striiformis urediniospores were obtained from the susceptible wheat cultivar Mingxian 169 in an experimental field (approximately $3 \mathrm{~m} \times 6 \mathrm{~m}$ area) at the Northwest $\mathrm{A} \& \mathrm{~F}$ University, Yangling, Shaanxi, China in the summer of 2015. Samples were collected and stored in a desiccator at $4^{\circ} \mathrm{C}$. The Puccinia striiformis species identity of these field samples were validated based on the complete internal transcribed spacer (ITS) sequence of ribosomal DNA (rDNA). Total genomic DNA was extracted from uredinospores of $P$. striiformis with CTAB method as described by Justesen et al. (2002). The ribosomal rDNAITS primers, ITS1 (5'-TCCGTAGGTGAACCTGCGG-3') and ITS4 (5'-TCCTCCGCTTATTGATATGC-3'), were synthesized by Sangon Bio-Tech Co., Ltd. Polymerase chain reaction (PCR) amplification was done following standard methods according to Zheng et al. (2017).

\section{Extraction of dsRNA}

DsRNA was extracted from $1.0 \mathrm{~g}$ of $P$. striiformis urediniospores according a described method with minor modifications (Zheng et al., 2014), and absorption column made up of cellulose powder CF-11 (Whatman, United Kingdom) was used. Extracted dsRNAs were treated with DNase I and S1 nuclease (TaKaRa Bio Inc) to remove genomic DNA and single-stranded RNA (ssRNA) contaminations, the qualities of which were then analyzed based on $1.0 \%(\mathrm{w} / \mathrm{v})$ agarose gel electrophoresis.

\section{cDNA Library Construction and Illumina Sequencing}

The dsRNA sample $(1.0 \mu \mathrm{g})$ was used for cDNA library construction using the NEBNext ${ }^{\circledR}$ Ultra $^{\mathrm{TM}}$ RNA Library Prep Kit (Illumina, United States) following manufacturer's instructions. The cDNA is end-repaired and adenylated prior to adaptor ligation, library construction and amplification under this method. Then the sequenced-ready library was subjected to 5 million of 150 nucleotide (nt) paired-end reads using Illumina HiSeq 4000 technology. The cDNA library construction and deep sequencing analysis were carried out by Shanghai Hanyu BioTech Co., Ltd. After deep sequencing (>40,000 × coverage), raw reads were cleaned by removing adapter sequences and lowquality bases (PHRED quality scores $\leq 5$ ), and truncated reads smaller than 35 bp were discard. 29 contigs were obtained and the N50 length is 1,489 nt. The total length of sequencing is about $37 \mathrm{~kb}$ with a GC content of $42.06 \%$. De novo assembly of contiguous sequences was conducted using the Velvet de novo assembly algorithm with k-mer: 69. Minimum contig length was $500 \mathrm{bp}$ as well as minimum coverage was 18 . The ends and the other parts of the sequences were all confirmed by Sanger sequencing. To obtain the termini of the dsRNAs, rapid amplification of cDNA ends (RACE) was performed (Zheng et al., 2013).

\section{Database Search and Sequence Analysis}

Open reading frames (ORFs) were identified using the National Center for Biotechnology Information (NCBI) ORF Finder program $^{1}$. Motif searches were performed in three databases, including $\mathrm{PROSITE}^{2}, \mathrm{Pfam}^{3}$ and $\mathrm{CDD}^{4}$. RNA pseudoknot structure was predicted using the DotKnot program and drawn with PseudoViewer3 (Byun and Han, 2009; Sperschneider and Datta, 2010).

\section{Phylogenetic Analysis}

Phylogenetic trees were constructed based on the deduced amino acid sequences of the putative RdRp and CP regions using the maximum-likelihood (ML) method of the MEGA program (version 6.0) with 1,000 bootstrap replicates as described

\footnotetext{
${ }^{1}$ http://www.bioinformatics.org/sms2/orf_find.html

${ }^{2}$ http://www.expasy.ch/

${ }^{3}$ http://pfam.sanger.ac.uk/

${ }^{4}$ http://www.ncbi.nlm.nih.gov/Structure/cdd/wrpsb.cgi
} 

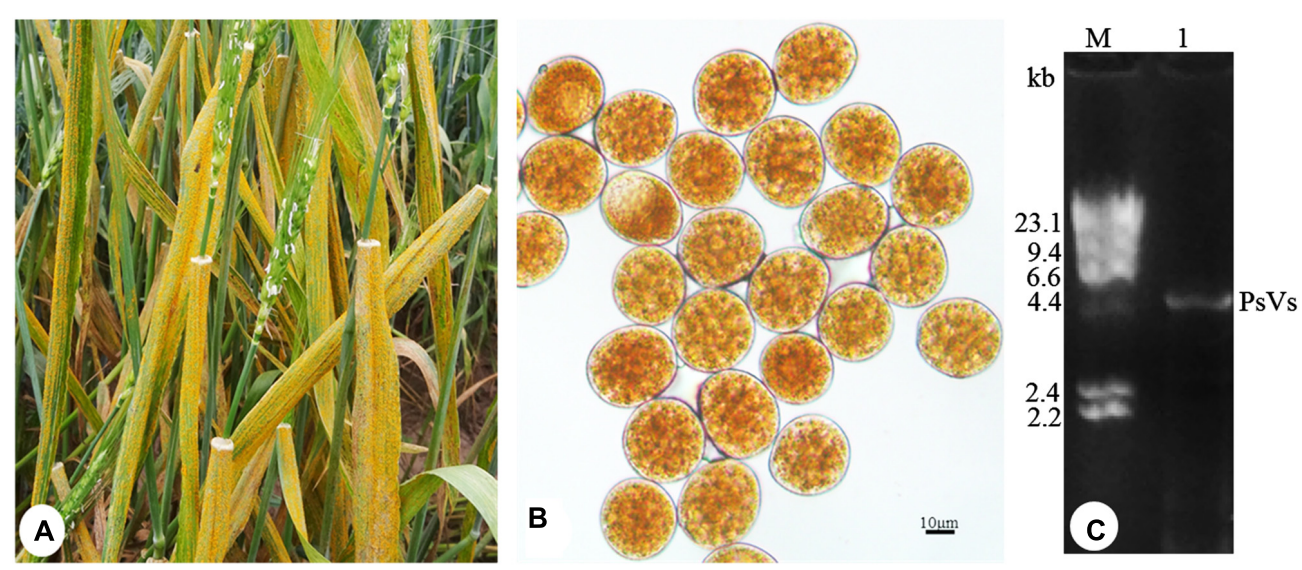

FIGURE 1 | Puccinia striiformis f. sp. tritici urediniospores contain dsRNA-like nucleic acids elements. (A) Symptoms of stripe rust disease on wheat leaves. (B) P. striiformis urediniospores collected from wheat leaves. (C) Agarose gel electrophoresis of dsRNA extracted from the urediniospores of $P$. striiformis. M indicates molecular markers of $\lambda$ DNA digested with Hind III.
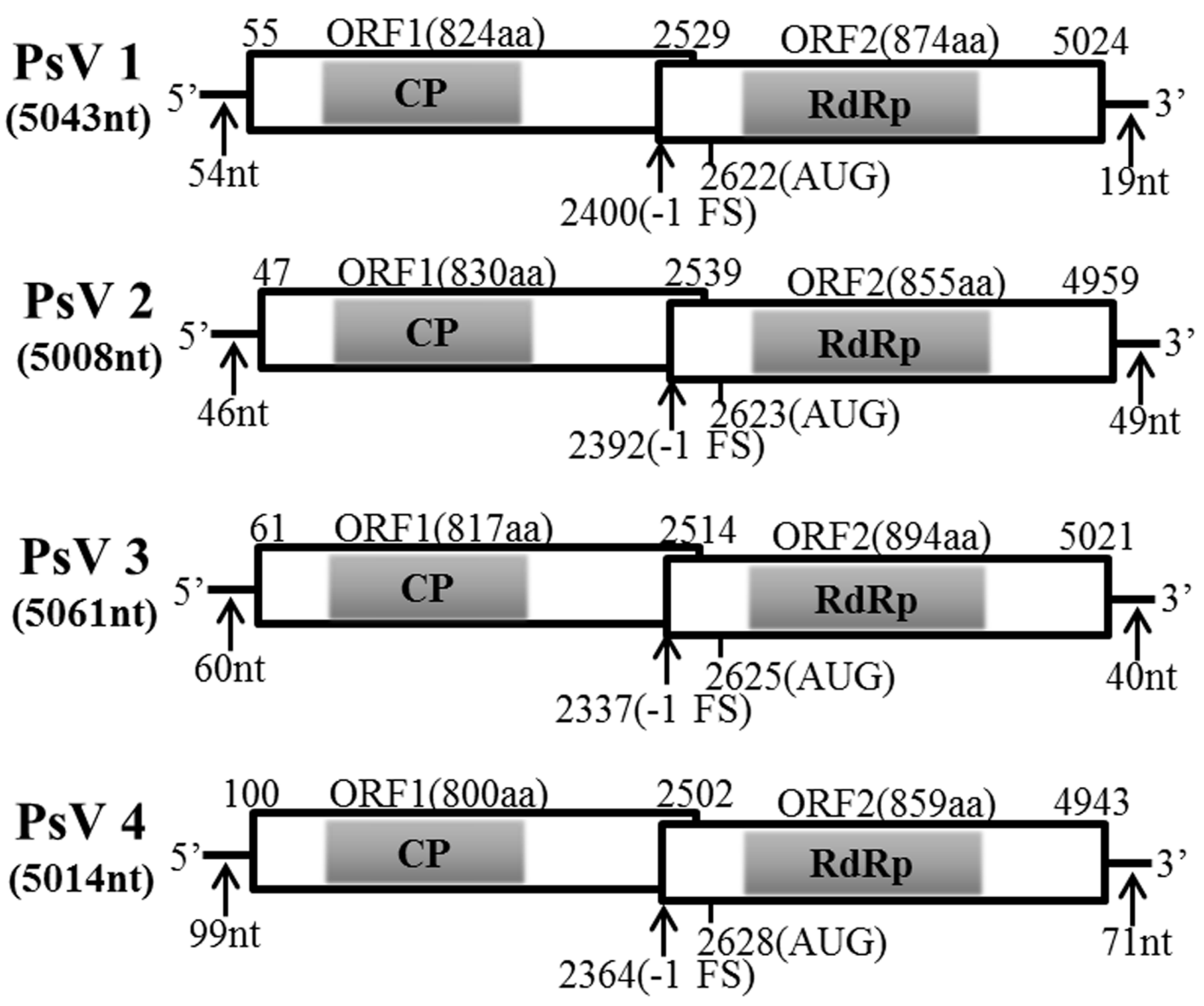

FIGURE 2 | Genomic organizations of PsVs sequences. The two overlapping ORFs and the untranslated regions (UTRs) are shown by open boxes and a single line, respectively. The conserved CPs and RdRps domain are indicated by shadows. Nucleotide positions of ORFs and the putative slippery site for -1 frameshifting are shown with black arrows. 


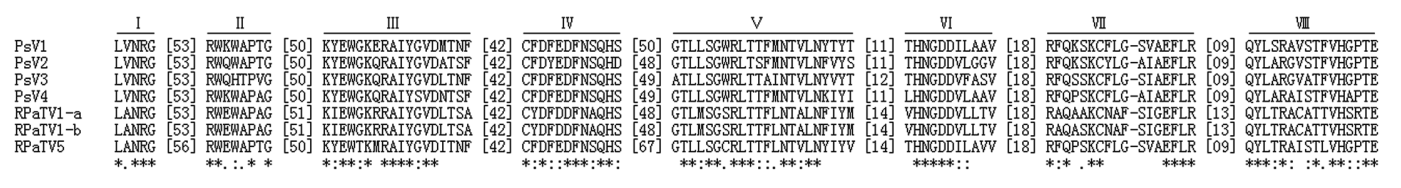

FIGURE 3 | Sequence alignment of PSV RdRp motifs with those of selected viruses in the genus Totivirus. Horizontal lines above the alignment indicate the eight motifs, numbers in brackets suggest the amino acid positions, shadow area and asterisks indicate identical amino acid residues, colons indicate the similar residues.

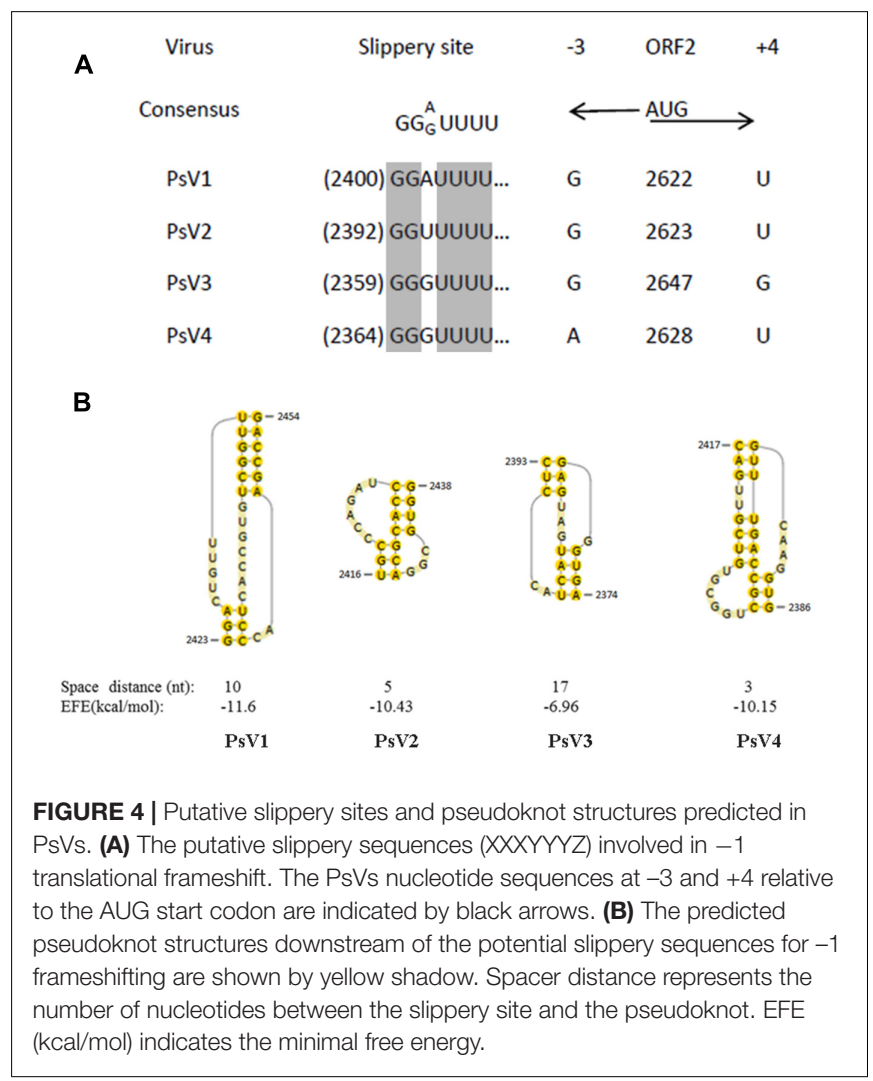

previously with minor modifications (Kondo et al., 2016). Multiple alignments of the sequences of RdRp and $\mathrm{CP}$ were conducted with Clustal-X program (Thompson et al., 1997).

\section{Purification of Viral Particles and Electron Microscopy}

Virus particles were purified using the method as described previously with minor modifications (Sanderlin and Ghabrial, 1978). Three gram P. striiformis urediniospores were grounded into fine powder in the presence of liquid nitrogen with a sterilized mortar and pestle. The powder was then mixed with $200 \mathrm{ml}$ extraction buffer made up of $0.1 \mathrm{M}$ sodium phosphate, and $\mathrm{pH} 7.0$ containing $3 \%$ Triton X-100. The suspension was centrifuged at $10,000 \times g$ for $20 \mathrm{~min}$ to remove the spore cell debris. Subsequently, the supernatant was subject to a $1.5 \mathrm{~h}$ ultracentrifugation at $120,000 \times g$ for viral particle precipitation. The pellets were then suspended in $0.1 \mathrm{M}$ sodium phosphate buffer and centrifuged at $15,000 \times g$ for $30 \mathrm{~min}$. Then the suspension containing the virus particles was fractionated via a

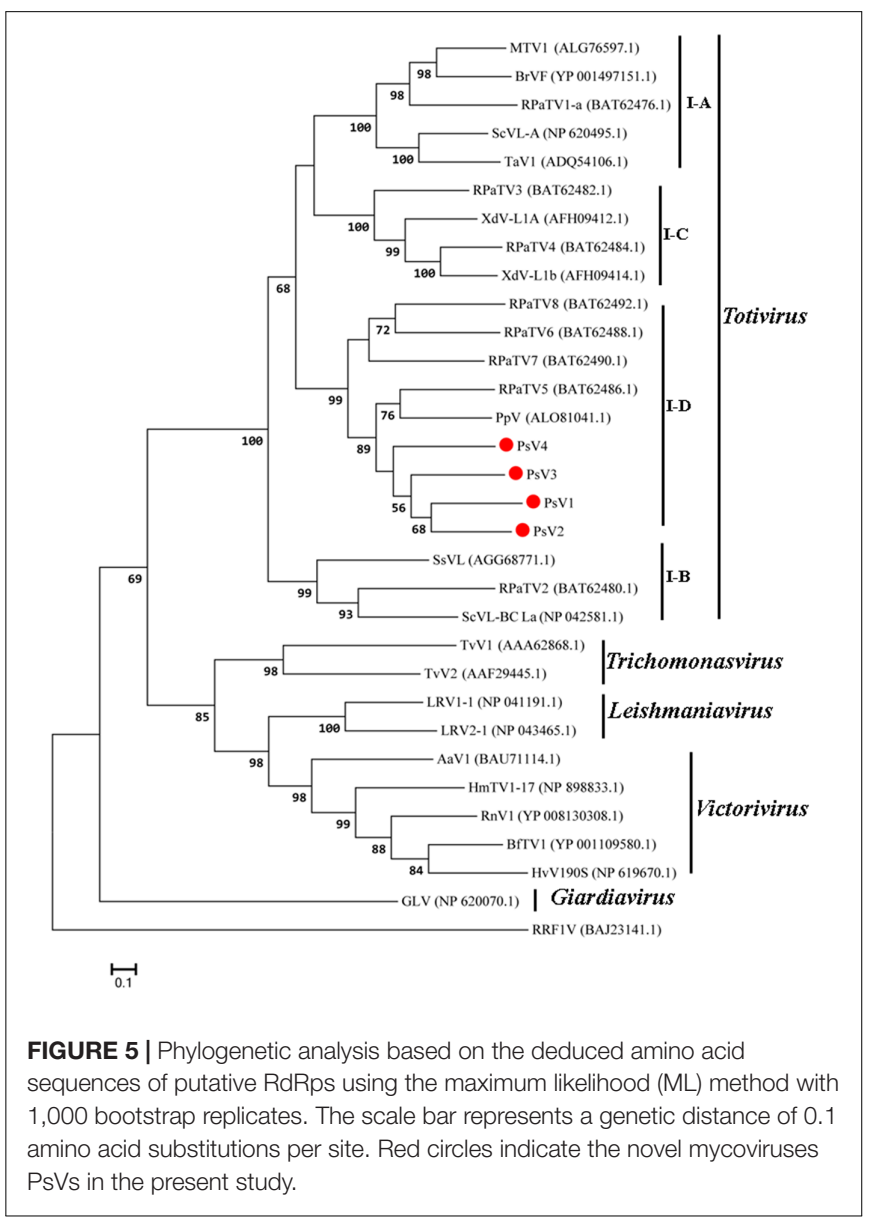

$10-40 \%(\mathrm{w} / \mathrm{v})$ sucrose gradient by centrifugation at $60,000 \times g$ for $3.5 \mathrm{~h}$. Fractions in the middle portion of the tube were collected, and stained with $2 \%$ phosphotungstic acid (pH 7.4) and observed under a transmission electron microscope (TEM) (HT7700, Japan).

\section{Validation of the Presence of Mycovirus-Like dsRNAs in Isolated Viral Particles}

The dsRNAs from virus particles was extracted using phenol, chloroform and isoamyl alcohol, and then subjected to electrophoresis in 1\% (w/v) agarose gel. Using the dsRNAs from virus particles as templates, complementary DNAs were synthesized as described by Xie et al. (2011) with tagged random dN6 primers (Rong et al., 2002; Zheng 


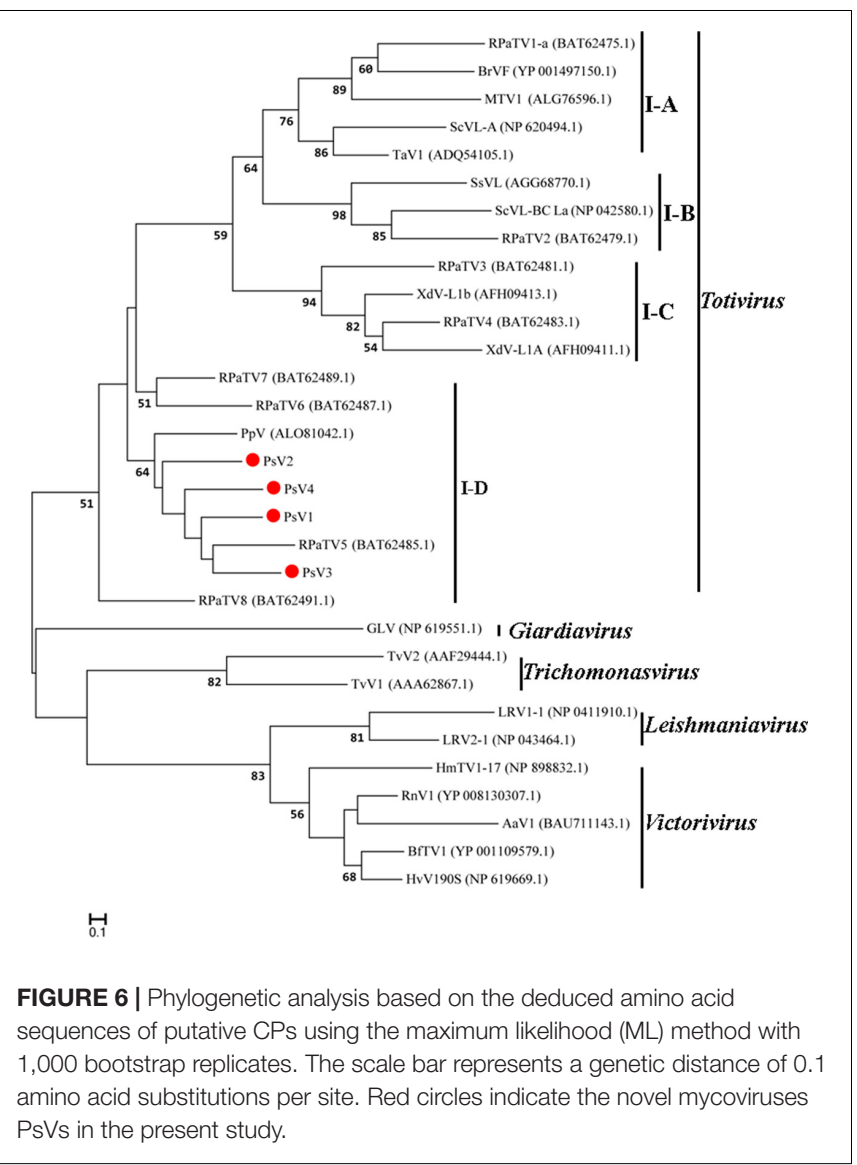

et al., 2013). Reverse transcription polymerase chain reaction (RT-PCR) was then performed with specific primers (Supplementary Table S1) which target the four PsVs respectively according to the method of Vainio et al. (2012) with minor modifications.

\section{RESULTS}

\section{$P$. strïformis Urediniospores Contain Putative Totiviruses-Like dsRNAs}

To detect dsRNA virus, $P$. striiformis urediniospores were collected from heavily infected wheat leaves growing in the field (Figure 1A). Urediniospores were round-shaped and approximately $30 \mu \mathrm{m}$ in diameter (Figure 1B). The sequence length of ITS is $663 \mathrm{bp}$ and it has 99\% sequence identity to the $P$. striiformis strain CYR32. The result confirmed the fungus belonged to $P$. striiformis species. Nucleic acid extraction obtained nucleic acid bands of approximately $5.0 \mathrm{~kb}$ in size (Figure 1C) that resisted DNase I and S1 nuclease digestions, indicating the presence of dsRNA-like nucleotides.

Four P. striiformis-derived totivirus-like sequences, termed as Puccinia striiformis virus 1 to 4 (PsV1 to 4) (Supplementary Table S2), were identified from the post-assembly contigs by deep sequencing. The $5^{\prime}$ - and $3^{\prime}$ - untranslated regions (UTRs) of all PsVs were obtained. The complete nucleotide sequences of
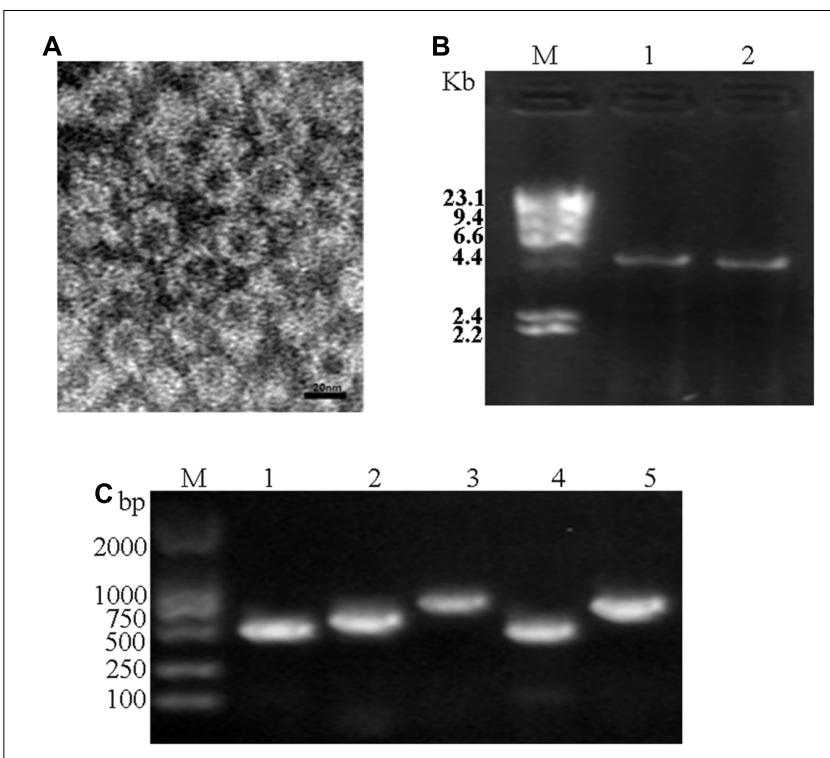

FIGURE 7 | Electron microscopy and RT-PCR. (A) Viral particles purified from field-collected $P$. striiformis urediniospores. (B) Agarose gel electrophoresis of dsRNAs extracted from purified virus particles of PsVs (lane 1) and the urediospores of $P$. striiformis from field-collected samples (lane 2).

(C) Confirmation of identity of dsRNAs extracted from purified virus particles using PsVs 1-4 specific primers (lanes 1-4, respectively) (Supplementary Table S1). M indicates molecular markers of $\lambda$ DNA digested with Hind III.

PsVs (1 to 4), ranging from 5,008 to 5,061 nt, were all deposited in GenBank under accession numbers KY207361-KY207364, respectively.

Pair-wise comparisons among proteins encoded by PsV1 to 4 revealed moderate levels $(37-42 \%)$ of amino acid sequence identity of RdRp, but low-level similarities (31-33\%) among CP. It is notable that the $5^{\prime}$-end sequences of PsVs 1 to 4 (5'-AUAAAUCCCC. . - $3^{\prime}, \quad 5^{\prime}$-AUACAAUCCCC. . - $3^{\prime}$, $5^{\prime}$-AUAUAACUCCC...-3' and 5'-AUAAAACCCCC...--3', respectively) appeared to be partially conserved. Likewise, the $3^{\prime}$-end sequences were not conserved (data not shown). All four genomes contained two ORFs encoding CP and RdRp respectively (ORF1 and ORF2) (Figure 2), such organization is typical of totiviruses. The predicted $\mathrm{CP}$ and $\mathrm{RdRp}$ proteins showed moderate levels of amino acid sequence identities (32-34\% for CP and 39-41\% for RdRp, respectively) with Phakopsora pachyrhizi mycovirus (PpV) and red clover powdery mildew-associated totivirus 5 (RPaTV5) (Supplementary Table S3). A search of conserved domain database (CDD) and multiple protein alignment confirmed that the predicted RdRp domains contain eight conserved motifs (I to VIII), including the GDD motif, which are the typical characteristics of mycovirual RdRps (Figure 3) (Routhier and Bruenn, 1998).

The PsV sequences between position -3 and +4 relative to the ORF2 AUG start codons (Figure 4A) are similar to the translation initiation sites of the viruses of plants and yeasts (Lütcke et al., 1987; Hamilton et al., 1987). Sequence analysis of PsVs indicated that there is an overlap region between ORFs 1 and 2 (Figure 2). It is therefore possible that ORFs 2 of 
PsVs is translated as a fusion protein with ORFs 1 through a -1 ribosomal frameshift which is a canonical slippery sites ' $\mathrm{XXXYYYZ'} \mathrm{(where} \mathrm{X}$ is $\mathrm{A} / \mathrm{C} / \mathrm{G} / \mathrm{U}, \mathrm{Y}$ is $\mathrm{A} / \mathrm{U}$, and $\mathrm{Z}$ is $\mathrm{A} / \mathrm{C} / \mathrm{U}$ ) within the overlapping region (Bekaert and Rousset, 2005). The slippery site 'GGA/GUUU' sequence in PsV1 to 4 sequences (Figure $4 \mathrm{~B}$ ) is similar to these of the RPaTVs (Kondo et al., 2016). Using the DotKnot program, a pseudoknot structure was predicted in the downstream of each putative slippery site (Figure 4B). RNA pseudoknots are known to help pausing the translating ribosome and increasing the frequency of frameshifting (Plant et al., 2003; Zhai et al., 2008; Kondo et al., 2016).

\section{Phylogenetic Analyses}

The maximum likelihood (ML) tree for RdRP is shown in Figure 5, Totivirus contained four subgroups, I-A, I-B, I-C and I-D. PsV1 to 4 clustered with RPaTVs 5 to 8 and PpV in the subgroup I-D. The ML phylogeny based on CPs is shown in Figure 6, which had similar topology as the one based on RdRPs.

Taken together, genome organizations, amino acid sequence alignments and phylogenetic analyses all support that PsV1 to 4 are new members of the genus Totivirus within the family Totiviridae.

\section{Observation of Viral Particle and Validation of the Viral Genome Sequences}

Under TEM, the viral particles purified from P. striiformis urediniospores were isometric with an average diameter of $35 \mathrm{~nm}$ (Figure 7A). The sizes of dsRNAs from viral particles were similar with the dsRNAs extracted directly from $P$. striiformis urediniospores (Figure 7B). Using the dsRNAs extracted from viral particles as templates, we performed RT-PCR based on PsVspecific primers targeting RdRps, which successfully amplified products and in all cases the sizes were identical to ones expected based on the PsV genomes (Figure 7C).

\section{DISCUSSION}

Characterization of newly isolated fungal viruses may contribute to understanding of the evolution and diversity of viruses (Chiba et al., 2009; Nibert et al., 2013; Zheng et al., 2014). Although mycoviruses have been identified from all major groups of filamentous fungi (Ghabrial and Suzuki, 2009), it is rarely reported in obligate biotrophs, such as rust fungi and powdery mildews most likely due to their unculturablity (Kondo et al., 2016). Recently, with the development of next generation sequencing technologies, new mycoviruses have been identified directly from field-collected fungal samples (Feldman et al., 2012; Kondo et al., 2016; Marzano and Domier, 2016). The present study revealed four complete (PsV1 to 4 ) totiviral sequences. The presence of these PsVs was further validated by TEM and RTPCR. To the best of our knowledge, this study provides the first evidence of dsRNA mycoviruses infections in the P. striiformis $\mathrm{f}$. sp. tritici, the causal agent of wheat stripe rust.
Each of the four PsV genomes contained two overlapping ORFs which encode the conserved domains of CP and RdRp, respectively. Moreover, the four PsVs were deduced to universally contain the -1 ribosomal frameshifting at the overlapping regions (Figure 2), which could facilitate translation of ORF1 and ORF2 as a fusion polyprotein. The predicted ORF2 coding strategy of PsVs were in line with members of genus Totivirus in the family Totiviridae, such as Saccharomyces cerevisiae virus L-A (ScVL-A) (Dinman et al., 1991) and red clover powdery mildew-associated totiviruses (RPaTVs) (Kondo et al., 2016).

Phylogenetic analysis with RdRp and CP sequences placed PsVs in a distinctive clade with members of Totivirus in the family Totiviridae (Figures 5, 6). Interestingly, both RdRp and CP phylogeny placed PsV1 to 4 together with RPaTVs 5 to 8 and $\mathrm{PpV}$ in the subgroup I-D. Interestingly, all the virus hosts in subgroup I-D are obligate biotrophic fungi, such as $P$. striiformis, $P$. pachyrhizi, and powdery mildew fungi, indicating an unknown interaction between obligate biotrophic fungi and totiviruses in this subgroup.

The current criteria for species demarcations of the genus Totivirus require less than $50 \%$ sequence identity of CP/RdRp proteins (Wickner et al., 2011). In the present study, proteins encoded by PsV1 to 4 shared moderate levels (RdRp, 37-42\%; $\mathrm{CP}, 31-33 \%$ ) of amino acid sequence identities to known totivirus species and among themselves, based on which they should represent novel totivirus species.

Taken together, this study characterized the molecular features of PsVs present in field-collected samples of wheat stripe rust fungus. The four novel viruses PsVs belong to the genus Totivirus in the family Totiviridae. This study demonstrates the presence of diverse, novel totiviruses in the P. striiformis $\mathrm{f}$. sp. tritici populations, characterizing their interactions with the $P$. striiformis host will potentially allow for developing novel rust disease control strategies.

\section{AUTHOR CONTRIBUTIONS}

ZK designed experiments; LZ performed the experiments; LZ, XL, XfL, and SJ analyzed the data; JZ, GZ, PL, and JW joined the discussion and gave the original ideas; $L Z$ wrote the paper.

\section{ACKNOWLEDGMENTS}

This work was supported by the National Key Basic Research Program of China (No. 2013CB127700), the China Postdoctoral Science Foundation (No. 2016M592845), the Shaanxi Postdoctoral Science Foundation (No. 2016BSHEDZZ116) and the National Natural Science Foundation of China (No. 31701732).

\section{SUPPLEMENTARY MATERIAL}

The Supplementary Material for this article can be found online at: https://www.frontiersin.org/articles/10.3389/fmicb. 2017.01960/full\#supplementary-material 


\section{REFERENCES}

Bekaert, M., and Rousset, J. P. (2005). An extended signal involved in eukaryotic -1 frameshifting operates through modification of the E site tRNA. Mol. Cell 17, 61-68. doi: 10.1016/j.molcel.2004.12.009

Byun, Y., and Han, K. (2009). Pseudoviewer 3: generating planar drawings of large-scale RNA structures with pseudoknots. Bioinformatics 25, 1435-1437. doi: 10.1093/bioinformatics/btp252

Chen, W. Q., Wu, L. R., Liu, T. G., Xu, S. C., Jin, S. L., Peng, Y. L., et al. (2009). Race dynamics, diversity, and virulence evolution in Puccinia striiformis $\mathrm{f}$. sp. tritici, the causal agent of wheat stripe rust in China from 2003 to 2007. Plant Dis. 93, 1093-1101. doi: 10.1094/PDIS-93-11-1093

Chen, X. M. (2005). Epidemiology and control of stripe rust [Puccinia striiformis f. sp. tritici] on wheat. Can. J. Plant Pathol. 27, 314-337. doi: 10.1080/ 07060660509507230

Chiba, S., Salaipeth, L., Lin, Y. H., Sasaki, A., Kanematsu, S., and Suzuki, N. (2009). A novel bipartite double-stranded RNA mycovirus from the white root rot fungus Rosellinia necatrix: molecular and biological characterization, taxonomic considerations, and potential for biological control. J. Virol. 83, 12801-12812. doi: 10.1128/JVI.01830-09

Cooper, B., Campbell, K. B., and Garrett, W. M. (2016). Expression of a synthetic rust fungal virus cDNA in yeast. Arch. Virol. 161, 111-123. doi: 10.1007/s00705015-2639-0

Dinman, J. D., Icho, T., and Wickner, R. B. (1991). A -1 ribosomal frameshift in a double-stranded RNA virus of yeast forms a gag-pol fusion protein. Proc. Natl. Acad. Sci. U.S.A. 88, 174-178. doi: 10.1073/pnas.88.1.174

Feldman, T. S., Morsy, M. R., and Roossinck, M. J. (2012). Are communities of microbial symbionts more diverse than communities of macrobial hosts? Fungal Biol. 116, 465-477. doi: 10.1016/j.funbio.2012.01.005

Ghabrial, S. A. (1998). Origin, adaptation and evolutionary pathways of fungal viruses. Virus Genes 16, 119-131. doi: 10.1023/A:100796622 9595

Ghabrial, S. A., Jiang, D., Nibert, M. L., and Suzuki, N. (2015). 50-plus years of fungal viruses. Virology 479, 356-368. doi: 10.1016/j.virol.2015. 02.034

Ghabrial, S. A., and Suzuki, N. (2009). Viruses of plant pathogenic fungi. Annu. Rev. Phytopathol. 47, 353-384. doi: 10.1146/annurev-phyto-080508081932

Goodman, R. P., Ghabrial, S. A., Fichorova, R. N., and Nibert, M. L. (2011). Trichomonasvirus: a new genus of protozoan viruses in the family Totiviridae. Arch. Virol. 156, 171-179. doi: 10.1007/s00705-0100832-8

Hamilton, R., Watanabe, C. K., and de Boer, H. A. (1987). Compilation and comparison of the sequence context around the AUG start codons in Saccharomyces cerevisiae mRNAs. Nucleic Acids Res. 15, 3581-3593. doi: 10. 1093/nar/15.8.3581

Justesen, A. F., Ridout, C. J., and Hovmøller, M. S. (2002). The recent history of Puccinia striiformis f. sp. tritici in Denmark as revealed by disease incidence and AFLP markers. Plant Pathol. 51, 13-23. doi: 10.1046/j.0032-0862.2001. 00651.x

Kondo, H., Hisano, S., Chiba, S., Maruyama, K., Andika, I. B., Toyoda, K., et al. (2016). Sequence and phylogenetic analyses of novel totivirus-like doublestranded RNAs from field-collected powdery mildew fungi. Virus Res. 213, 353-364. doi: 10.1016/j.virusres.2015.11.015

Lin, Y. H., Chiba, S., Tani, A., Kondo, H., Sasaki, A., Kanematsu, S., et al. (2012). A novel quadripartite dsRNA virus isolated from a phytopathogenic filamentous fungus, Rosellinia necatrix. Virology 426, 42-50. doi: 10.1016/j.virol. 2012.01.013

Link, T. I., Lang, P., Scheffler, B. E., Duke, M. V., Graham, M. A., Cooper, B., et al. (2014). The haustorial transcriptomes of Uromyces appendiculatus and Phakopsora pachyrhizi and their candidate effector families. Mol. Plant Pathol. 15, 379-393. doi: 10.1111/mpp.12099

Lütcke, H. A., Chow, K. C., Mickel, F. S., Moss, K. A., Kern, H. F., and Scheele, G. A. (1987). Selection of AUG initiation codons differs in plants and animals. EMBO J. 6, 43-48.

Marzano, S. Y., and Domier, L. L. (2016). Novel mycoviruses discovered from metatranscriptomics survey of soybean phyllosphere phytobiomes. Virus Res. 213, 332-342. doi: 10.1016/j.virusres.2015.11.002
Newton, A. C., Caten, C. E., and Johnson, R. (1985). Variation for isozymes and double-stranded RNA among isolates of Puccinia striiformis and two other cereal rusts. Plant Pathol. 34, 235-247. doi: 10.1111/j.1365-3059.1985. tb01355.x

Nibert, M. L., Tang, J., Xie, J., Collier, A. R., Ghabrial, S. A., Baker, T. S., et al. (2013). 3D structures of fungal partitiviruses. Adv. Virus Res. 86, 59-85. doi: 10.1016/B978-0-12-394315-6.00003-9

Nuss, D. L. (2005). Mycoviruses at the fungal-plant interface. Nat. Rev. Microbiol. 3, 632-642. doi: 10.1038/nrmicro1206

Pearson, M. N., Beever, R. E., Boine, B., and Arthur, K. (2009). Mycoviruses of filamentous fungi and their relevance to plant pathology. Mol. Plant Pathol. 10, 115-128. doi: 10.1111/j.1364-3703.2008.00503.x

Plant, E. P., Jacobs, K. L., Harger, J. W., Meskauskas, A., Jacobs, J. L., Baxter, J. L., et al. (2003). The 9-A solution: how mRNA pseudoknots promote efficient programmed -1 ribosomal frameshifting. RNA 9, 168-174. doi: 10.1261/rna. 2132503

Rong, R., Rao, S., Scott, S. W., Carner, G. R., and Tainter, F. H. (2002). Complete sequence of the genome of two dsRNA viruses from Discula destructive. Virus Res. 90, 217-224. doi: 10.1016/S0168-1702(02)00178-8

Routhier, E., and Bruenn, J. A. (1998). Functions of conserved motifs in the RNAdependent RNA polymerase of a yeast double-stranded RNA virus. J. Virol. 72, 4427-4429.

Sanderlin, R. S., and Ghabrial, S. A. (1978). Physicochemical properties of two distinct types of virus-like particles from Helminthosporium victoriae. Virology 87, 142-151. doi: 10.1016/0042-6822(78)90166-6

Sperschneider, J., and Datta, D. (2010). Dotknot: pseudoknot prediction using the probability dot plot under a refined energy model. Nucleic Acids Res. 38:e103. doi: 10.1093/nar/gkq021

Thompson, J. D., Gibson, T. J., Plewniak, F., Jeanmougin, F., and Higgins, D. G. (1997). The CLUSTAL_X windows interface: flexible strategies for multiple sequence alignment aided by quality analysis tools. Nucleic Acids Res. 25, 4876-4882. doi: 10.1093/nar/25.24.4876

Vainio, E. J., Hyder, R., Aday, G., Hansen, E., Piri, T., Doğmus̨-Lehtijärvi, T., et al. (2012). Population structure of a novel putative mycovirus infecting the conifer root-rot fungus Heterobasidion annosum sensu lato. Virology 422, 366-376. doi: 10.1016/j.virol.2011.10.032

Wellings, C. R. (2011). Global status of stripe rust: a review of historical and current threats. Euphytica 179, 129-141. doi: 10.1007/s10681-0110360-y

Wickner, R. B., Ghabrial, S. A., Nibert, M. L., Patterson, J. L., and Wang, C. C. (2011). "Family Totiviridae," in Virus Taxonomy: Classification and Nomenclature of Viruses: Ninth Report of the International Committee on Taxonomy of Viruses, eds A. M. Q. King, M. J. Adams, E. B. Carstens, and E. J. Lefkowits (Tokyo: Elsevier Academic Press), 639-650.

Xie, J., Xiao, X., Fu, Y., Liu, H., Cheng, J., Ghabrial, S. A., et al. (2011). A novel mycovirus closely related to hypoviruses that infects the plant pathogenic fungus Sclerotinia sclerotiorum. Virology 418, 49-56. doi: 10.1016/j.virol.2011. 07.008

Yu, X., Li, B., Fu, Y. P., Xie, J. T., Cheng, J. S., Ghabrial, S. A., et al. (2013). Extracellular transmission of a DNA mycovirus and its use as a nature fungicide. Proc. Natl. Acad. Sci. U.S.A. 110, 1452-1457. doi: 10.1073/pnas.12137 55110

Zhai, Y. G., Lv, X. J., Sun, X. H., Fu, S. H., Gong, Z. D., Fen, Y., et al. (2008). Isolation and characterization of the full coding sequence of a novel densovirus from the mosquito Culex pipiens pallens. J. Gen. Virol. 89, 195-199. doi: 10.1099/vir.0. 83221-0

Zhang, R., Qiu, B., Tien, P., and Pryor, A. (1994). Presence of double-stranded RNA in the virus-like particles of Uromyces vignae Barcl. Acta Mycol. Sin. 13, 48-51.

Zheng, L., Liu, H. Q., Zhang, M. L., Cao, X., and Zhou, E. X. (2013). The complete genomic sequence of a novel mycovirus from Rhizoctonia solani, AG-1 IA strain B275. Arch. Virol. 158, 1609-1612. doi: 10.1007/s00705-0131637-3

Zheng, L., Zhang, M. L., Chen, Q. G., Zhu, M. H., and Zhou, E. X. (2014). A novel mycovirus closely related to viruses in the genus Alphapartitivirus, confers hypovirulence in the phytopathogenic fungus Rhizoctonia solani. Virology 456, 220-226. doi: 10.1016/j.virol.2014.03.029

Zheng, L., Zhao, J., Liang, X. F., Zhan, G. M., Jiang, S. C., and Kang, Z. S. (2017). Identification of a novel Alternaria alternata strain able 
to hyperparasitize Puccinia striiformis f. sp. tritici, the causal agent of wheat stripe rust. Front. Microbiol. 8:71. doi: 10.3389/fmicb.2017. 00071

Conflict of Interest Statement: The authors declare that the research was conducted in the absence of any commercial or financial relationships that could be construed as a potential conflict of interest.
Copyright $\odot 2017$ Zheng, Lu, Liang, Jiang, Zhao, Zhan, Liu, Wu and Kang. This is an open-access article distributed under the terms of the Creative Commons Attribution License (CC BY). The use, distribution or reproduction in other forums is permitted, provided the original author(s) or licensor are credited and that the original publication in this journal is cited, in accordance with accepted academic practice. No use, distribution or reproduction is permitted which does not comply with these terms. 